This item was submitted to Loughborough's Research Repository by the author.

Items in Figshare are protected by copyright, with all rights reserved, unless otherwise indicated.

\title{
Influence of asymmetric valve strategy on large-scale and turbulent in- cylinder flows
}

\section{PLEASE CITE THE PUBLISHED VERSION}

https://doi.org/10.1177/1468087417725232

\section{PUBLISHER}

(C) The authors. Published by SAGE Journals

\section{VERSION}

AM (Accepted Manuscript)

\section{PUBLISHER STATEMENT}

This work is made available according to the conditions of the Creative Commons Attribution-NonCommercialNoDerivatives 4.0 International (CC BY-NC-ND 4.0) licence. Full details of this licence are available at: https://creativecommons.org/licenses/by-nc-nd/4.0/

\section{LICENCE}

CC BY-NC-ND 4.0

\section{REPOSITORY RECORD}

Butcher, Daniel, Adrian Spencer, and Rui Chen. 2017. "Influence of Asymmetric Valve Strategy on Large-scale and Turbulent In-cylinder Flows". figshare. https://hdl.handle.net/2134/25908. 


\title{
Influence of asymmetric valve strategy on large-scale and turbulent in-cylinder flows
}

\author{
Daniel Butcher ${ }^{1}$, Adrian Spencer ${ }^{1}$ and Rui Chen ${ }^{1}$
}

\section{@SAGE}

\begin{abstract}
Phase-locked particle imaging velocimetry (PIV) measurements are carried out in a direct-injected spark-ignition (DISI) single cylinder optical research engine equipped with fully variable valve timing (FVVT) to assess the impact of asymmetric intake valve lift strategies on the in-cylinder flow. The engine was operated under a range of asymmetric strategies, with one valve following a full lift profile, while the second intake valve is scaled as a factor of the first, expressed as \% maximum valve lift (MVL). Proper orthogonal decomposition (POD) combined with a proposed methodology allows instantaneous velocity fields to be decomposed into what are nominally demonstrated as coherent and turbulent constituent velocity fields. Analysis of the coherent fields reveals the behaviour of large scale structures within the flow, subject to cyclic variation. In the case of $40 \% \mathrm{MVL}$, an increase in the flow cyclic variability is observed. This is found to be as a result of a switch between a flow dominated by a counter-rotating vortex pair and a single vortex. The impact of MVL on the bulk motion is further evident by an increase in the magnitude of swirl ratio from 0.5 to -6.0 (at $75^{\circ} \mathrm{CA}$ ). Analysis of the turbulent constituent shows how increased valve life asymmetry leads to increased turbulence during the intake stroke by over $250 \%$. Finally, it is shown how the ensemble turbulence statistics may be misleading as stochastic fluctuations were found to be typically $66 \%$ of the total TKE calculated from the ensemble statistic in the tested conditions.
\end{abstract}

\section{Keywords}

Asymmetric valve strategy, internal combustion engine, cycle-to-cycle variation, turbulence, turbulent kinetic energy, particle image velocimetry, proper orthogonal decomposition

\section{Introduction}

In modern internal combustion engines (ICE), flow is characterised by both large scale, bulk motion and small scale turbulent motions. The determination and understanding of both of these contributions is vital in the development of current and future ICEs.

Large scale motion, both swirling and tumble motion are sought in many engine designs to assist in the transport of fuel, ensuring proper distribution. The large scale motion also provides insight into the cycle-to-cycle variation present in the flow ${ }^{1}$, known to have a negative impact on combustion stability. Conversely, an increase in the turbulent kinetic energy may enhance the mixing and combustion rate.

In this study, phase-locked in-cylinder flow velocities in an optically accessible, direct-injected spark-ignition (DISI) engine are measured using particle image velocimetry (PIV) under a range of valve timing strategies during the intake stroke. Specifically, measurements are taken at $75^{\circ}, 80^{\circ}$ and $85^{\circ}$ as these are correspond approximately to common injection timing in GDi engines. This timing is also the time of maximum piston speed and thus maximum airflow rate where the structures resulting from valve strategy are expected to be most pronounced. The resulting velocity fields are separated into coherent and turbulent constituent fields and analysed respectively to study the effects of valve strategy on both the large scale, coherent motions and the turbulent kinetic energy in the flow.

By successful separation of the constituent flow fields, it is possible to calculate more representative statistics and

\footnotetext{
${ }^{1}$ Department of Aeronautical and Automotive Engineering, Loughborough University

\section{Corresponding author:}

Daniel Butcher, Department of Aeronautical and Automotive Engineering, Loughborough University, Loughborough, Leicestershire, LE11 3TU, United Kingdom

Email: D.Butcher@lboro.ac.uk
} 
distributions about both the large scale motions and the turbulent kinetic energy.

Traditional Reynolds decomposition, Equation 1, is often used to in the analysis of flow by providing an average flow field and a fluctuating field where $\theta$ is crank angle in phaselocked measurements and $i$ is sample number. But it cannot distinguish between cyclic variations of large scale motion and turbulent fluctuations. Stone ${ }^{2}$ instead shows how the fields may be decomposed to two fluctuating components, a low frequency component, representing cyclic variation, $U^{*}$ and a high frequency component representing turbulence, $u^{\prime}$ as shown in Equation 2. Stone goes on to explain how the turbulent kinetic energy may be over-estimated by as much as $300 \%$ without this distinction between fluctuation contribution. In the presented work, TKE is calculated according to Equation 3, this is an estimate of the TKE, derived from 2-dimensional data where subscripts $1 \& 2$ denote the two components of velocity in the measured plane.

$$
\begin{gathered}
U(\theta, i)=\bar{U}(\theta)+U^{\prime}(\theta, i) \\
U(\theta, i)=\bar{U}(\theta)+U^{*}(\theta, i)+u^{\prime}(\theta, i) \\
T K E=\frac{1}{2}\left({\overline{U^{\prime}}}_{1}^{2}+{\overline{U^{\prime}}}_{2}^{2}\right)
\end{gathered}
$$

Several methods have been suggested to decompose the fluctuating velocity fields into a low-frequency fluctuation (representative of cyclic variation) and high-frequency fluctuation (representative of turbulence). Spatial filtering techniques based on fast Fourier transform (FFT) has been used in studies ${ }^{3-6}$. Olçmen et. al. ${ }^{7}$ gives a comprehensive comparison of methods including ensemble averaging, wavelet decomposition, proper orthogonal decomposition (POD) and combinations of these methods. The work concludes that a method involving POD yielded the best results.

POD, also known as the Karhunen-Loève decomposition or principle component analysis is a tool of probability theory used for modal decomposition of an ensemble of values or signals. It is has been applied in a wide range of disciplines including variable analysis and image processing (for references, see Berkooz et. al. ${ }^{8}$ ).

The technique was introduced in the field of turbulent flows by Lumley in 1967 and was used to separate large eddies in shear flows ${ }^{9}$. Since then, numerous studies have made use of POD methodology to study the flow fields in the ICE ${ }^{1 ; 10-14}$, particularly in the study of flow cyclic variations.

In the presented context, the POD technique decomposes time dependant velocity fields, $u\left(x, t_{i}\right)$ into a set of spatial modes, $\varphi^{(k)}(x)$ and temporal modes $a^{(k)}\left(t_{i}\right)$ according to Equation 4 where there exists $M$ velocity fields.

$$
u\left(x, t_{i}\right)=\sum_{k=1}^{M} a_{k}\left(t_{i}\right) \varphi_{k}(x) \quad i=1, \ldots, N
$$

In the determination of $\varphi(x)$ and $a\left(t_{i}\right)$, the classical method $^{15 ; 16}$ is often considered too computationally expensive. Instead, the 'method of snapshots' is commonly used ${ }^{17}$ and this methodology is utilized in the presented work.

Commonly, a truncation order is chosen based on the total energy content represented by the modes, for example $90 \%$ is used in the work by Graftieaux et. al. ${ }^{18}$. The issue of choosing an arbitrary value of energy representation is that in the ICE, there are two main contributors to flow fluctuation; cyclic variation and turbulence. In highly turbulent flows, there is no clear step-change in POD mode energy, making it difficult to define a cut-off value. Instead, the method used in this study exploits the expected correlation between the POD modes that are representative of the coherent structures and the expected lack of correlation between those representative of stochastic processes.

Using standard statistical approaches it is possible to determine the ensemble mean, $\bar{U}(\theta, i)$, in Equation 2 of the individual realisations, $U(\theta, i)$, by averaging over all i. Subtracting this ensemble mean from the individual realisations leaves the fluctuating components $U^{*}(\theta, i)$ and $u^{\prime}(\theta, i)$. POD analysis is then performed on the sum of these fluctuating velocity components, which by definition has a zero ensemble mean. Since POD does not produce individual modes, $\phi_{k}(x)$, that explicitly belong to each of the two fluctuating categories (cyclic variation or stochastic turbulence) this paper proposes a route to determining this in a prescribed analytical way as described in Equation 5 where $L \subset K$ identified as cyclic variation and $J$ are the remaining modes of $\mathrm{K}$.

$$
U^{*}(\theta, i)+u^{\prime}(\theta, i)=\sum_{L} a_{k}\left(t_{i}\right) \cdot \varphi_{k}(x)+\sum_{J} a_{k}\left(t_{i}\right) \cdot \varphi_{k}(x)
$$

\section{Experimental methodology}

\section{Optical engine and test conditions}

The engine used for PIV experiments in the presented work was the Lotus DISI single cylinder optical research engine (SCORE), Figure 1. The engine features an extended, bifurcated piston with a sapphire window and a full length fused silica liner providing optical access to both the swirl 


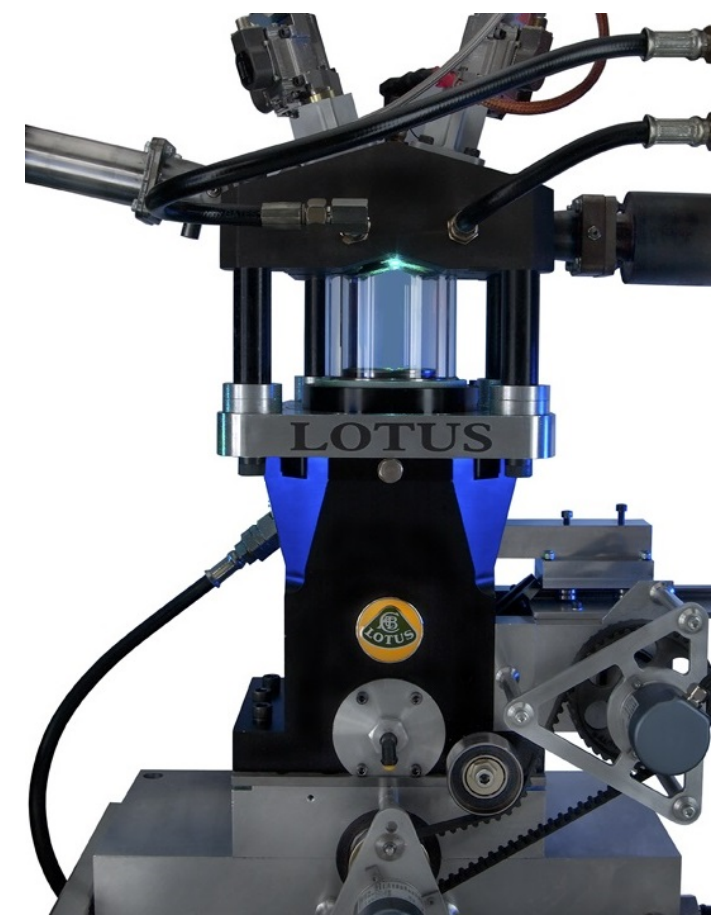

Figure 1. Optically accessible engine - Lotus SCORE

Table 1. Engine specifications

\begin{tabular}{cc}
\hline Bore & $88.0 \mathrm{~mm}$ \\
Stroke & $82.1 \mathrm{~mm}$ \\
Swept volume & $0.5 \mathrm{~L}$ \\
Compression ratio & $10: 1$ \\
Intake valve diameter & $31 \mathrm{~mm}$ \\
Exhaust valve diameter & $26 \mathrm{~mm}$ \\
Piston window diameter & $60 \mathrm{~mm}(52 \mathrm{~mm}$ avail. $)$ \\
Intake open / close / max. lift & $-15^{\circ} / 225^{\circ} / 9.35 \mathrm{~mm}$ \\
Exhaust open / close / max. lift & $495^{\circ} / 15^{\circ} / 9.35 \mathrm{~mm}$ \\
\hline
\end{tabular}

(horizontal) and tumble (vertical) planes. The engine is a four-valve, pent roof design with symmetrical ports and a flat top piston. The engine is capable of being motored or operated under fired or skip-fired conditions up to 5000 RPM. The main specifications are given in Table 1 where $0^{\circ}$ refers to TDC intake stroke. It should be noted that whilst the ports are symmetrical and fed from a plenum, the feed to the plenum may create a small level of flow asymmetry at the port.

Asymmetric valve strategies are realized through the use of a fully variable valve timing (FVVT) system, using electro-hydraulic valve actuation. The system, developed by Lotus is known as the active valve train (AVT). Throughout the presented work, the exhaust valve strategy is held unchanged according to Table 1 . The intake valve strategy is varied by keeping one of the valves' timings unchanged, while scaling the maximum valve lift (MVL) of the other valve by $0 \%, 20 \%, 40 \%, 60 \%, 80 \%$ and $100 \%$, maintaining opening and closing times as illustrated in Figure 2.

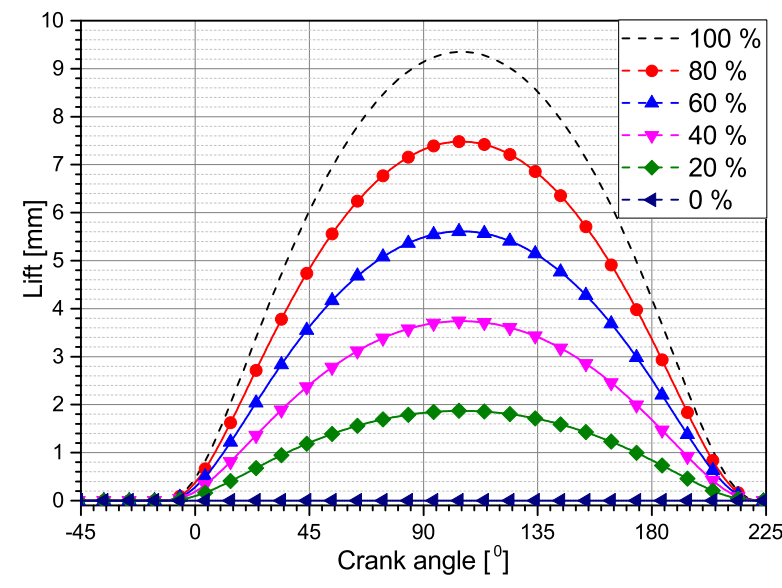

Figure 2. Intake valve schedules for asymmetric operation (dashed shows full valve lift)

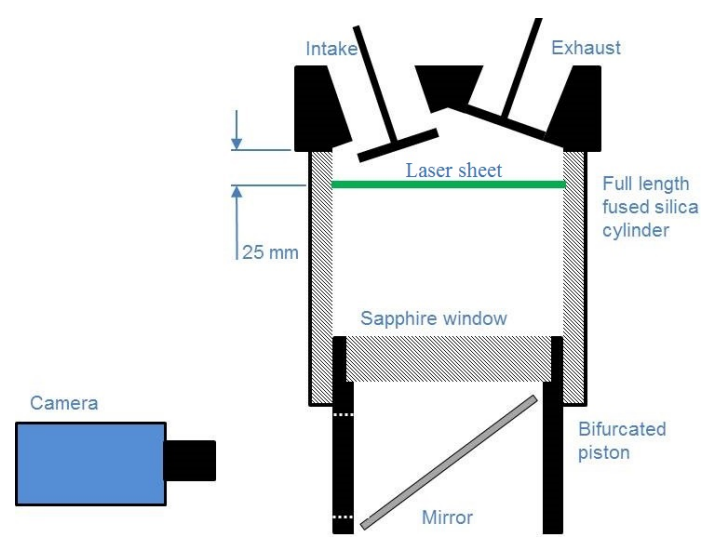

Figure 3. Schematic of optical engine for PIV measurements

For all test conditions, the engine is motored at 2000 RPM with a manifold pressure of 450 mbar (+/- 15 mbar). The resulting peak in-cylinder pressure is 8.0 bar $(+/-0.1$ bar) in all conditions, and is approximately equivalent to the air charge at a load of 3.0 bar IMEP under stoichiometric GDI operation. At the tested speed and load condition, the reduction in MVL was found to have no effect on the trapped mass, and required no alteration of the throttle plate angle. The intake flow rate and air temperature were not controlled during experiments, but were monitored throughout and found to be consistent between tests.

\section{PIV measurements}

A LaVision Flowmaster PIV system was employed throughout this study, comprising of a New Wave Solo Nd:YAG pulsed laser with associated sheet-forming optics, a Flowmaster 3S CCD camera fitted with a Nikkor $60 \mathrm{~mm}$ lens and the DaVis v8.2 software and programmable timing unit (PTU9). The positioning of equipment is illustrated in Figure 3, note the laser is not displayed as it is orthogonal, with the direction out of the image. 
Table 2. PIV inter-frame time, $\delta \mathrm{t}$

\begin{tabular}{cc}
\hline MVL scale & $\delta \mathrm{t}[\mu \mathrm{s}]$ \\
\hline 0 & 6 \\
20 & 8 \\
40 & 9 \\
60 & 10 \\
80 & 15 \\
100 & 15 \\
\hline
\end{tabular}

Seeding is introduced to the intake flow upstream of the intake plenum using the LaVision aerosol generator ${ }^{19}$. The seeder is is filled with olive oil of approximately $900 \mathrm{~kg} / \mathrm{m}^{3}$ density and produces droplets with mean diameter of $1 \mu \mathrm{m}$. This is suggested by Melling ${ }^{20}$ to be capable of following flows up to $10 \mathrm{kHz}$ and is therefore suitable for the work presented.

The New Wave Solo PIV laser used as the light source in the presented experiments has a maximum pulse energy of $120 \mathrm{~mJ} /$ pulse up to $15 \mathrm{~Hz}$ at the second harmonic frequency (532 nm) with a pulse duration of 3-5 ns. Throughout the presented work, the laser was operated at $70 \%$ power ( $84 \mathrm{~mJ} /$ pulse). The $6 \mathrm{~mm}$ diameter laser beam is shaped by sheet optics with a focal length of $-20 \mathrm{~mm}\left(10^{\circ}\right.$ angle of divergence) to provide a $1.5 \mathrm{~mm}$ thick sheet of sufficient width to fill the bore of the cylinder, ensuring a uniform light distribution within the measurement area. The sheet is kept in a constant location of $25 \mathrm{~mm}$ below the bottom of the pent roof, regardless of piston position. The earliest phase that may be measured in this configuration without interference from the piston is $75^{\circ} \mathrm{CA}$.

Image acquisition is via the LaVision Flowmaster 3S CCD camera which has an image resolution of $1280 \times 1024$ and pixel size of $6.7 \mu \mathrm{m} \times 6.7 \mu \mathrm{m}$. The camera was fitted with a Nikkor $60 \mathrm{~mm}$ macro lens, with the f-stop set to 11 . This was set to achieve a particle image diameter of 2-3 pixels (calculated according to Adrian \& Westerwheel ${ }^{21}$ ), sufficient to ensure there was no peak locking effect in the captured velocity fields. This was assessed within the DaVis software during preliminary set-up runs. During the preliminary testing, particle travel through the plane thickness was found to increase with reducing MVL, therefore the inter-frame time, $\delta \mathrm{t}$ was adjusted to account for this. By reducing the inter-frame time, the maximum particle displacement was also maintained at approximately $7-10$ pixels, which is suitable for an interrogation region of $32 \times 32$. The relevant values are presented in Table 2 . The captured field of view is represented in Figure 4.

At each valve condition, 800 cycle resolved measurements were taken at several crank-angle timings during the intake stroke; $75^{\circ}, 80^{\circ}, 85^{\circ} \mathrm{CA}$. Each valve condition

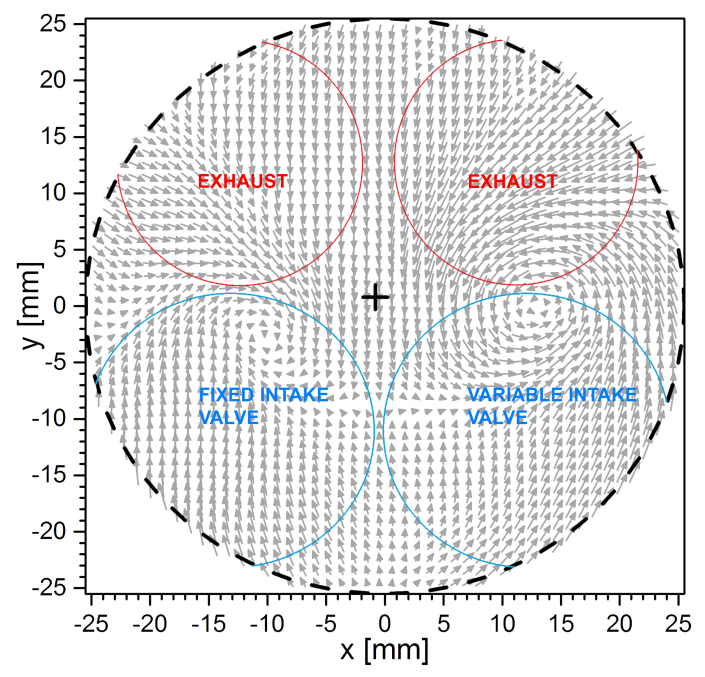

Figure 4. Captured field of view, approximate valve and spark locations indicated (Vectors from Figure 5(a) superimposed for orientation)

and measurement time necessitated a separate engine run to allow cleaning of and prevent fouling of the optical components.

The PIV processing, all of which was carried out in DaVis v8.2 software, included a pre-processing step where the images had a sliding background subtraction. This was found to be the most suitable solution to remove background and liner glare while retaining particle locations. This technique is particularly suited to the optical engine application as the glare from the liner changes throughout the engine run due to fouling from the piston rings.

The velocity vectors are obtained from multi-pass, decreasing size cross-correlation of each image pair. A single initial pass using an interrogation region of $64 \times 64$ with $50 \%$ overlap is followed by two further passes with a $32 \times 32$ pixel interrogation region with $87 \%$ overlap. An iterative median filter is applied to remove spurious vectors that are different to neighbouring vectors by more than 2 standard deviations. Vectors with a $\mathrm{Q}$ ratio of less than 1.3 are also rejected. On average, each vector field was found to contain fewer than $2 \%$ spurious vectors, in which case the vector is replaced either with the next choice vector or linearly interpolated from the neighbouring vectors.

Using a $32 \times 32$ interrogation region with the described image acquisition set-up allowed a spatial resolution of 2.2 $\mathrm{mm} \times 2.2 \mathrm{~mm}$. While this is not sufficient to resolve the smallest scale turbulence, it allows resolution of the expected integral length scale which is typically of the order of 4 $\mathrm{mm}^{2 ; 22 ; 23}$. Note, while this is the resolution of the recorded vector fields, for the purposes of clarity, the vector density has been reduced only for presentation. A summary of measurement parameters are given in Table 3. 
Table 3. PIV measurement parameters summary

\begin{tabular}{c|c}
\hline $\begin{array}{c}\text { Seeding } \\
\text { Type } \\
\text { Density }\end{array}$ & $\begin{array}{c}\text { Olive oil } \\
900 \mathrm{~kg} / \mathrm{m}^{3}\end{array}$ \\
Mean diameter & $1 \mu \mathrm{m}\left(\right.$ reference $\left.^{19}\right)$ \\
\hline Image capture & $60 \mathrm{~mm}$ \\
Lens focal length & $86 \mathrm{~mm} \times 69 \mathrm{~mm}$ \\
Field of view size & $69 \mu \mathrm{m} / \mathrm{px}$ \\
Spatial resolution & 0.1 \\
Magnification & $5.45 \mathrm{~mm}\left(\mathrm{f}_{\#=11)}\right.$ \\
Aperture & $6.7 \mu \mathrm{m} \times 6.7 \mu \mathrm{m}$ \\
CCD pixel size & $64 \times 64 \mathrm{initial}(50 \%$ overlap) \\
Interrogation region size & $2 \times 32 \times 32$ final $(87 \%$ overlap) \\
Particle image size & $2.3 \mathrm{px}$ \\
Particle density & $30-40 \mathrm{per} \mathrm{region}$ \\
Number of samples & $800 \mathrm{pairs}$ \\
\hline Illumination & \\
Wavelength & $532 \mathrm{~nm}$ \\
Repetition rate & $3.4 \mathrm{~Hz}$ \\
Sheet thickness & $1.5 \mathrm{~mm}$ \\
Pulse energy & $84 \mathrm{~mJ} / \mathrm{pulse}$ \\
\hline
\end{tabular}

When assessing the accuracy of the presented measurements there are a number of sources of uncertainty that must be considered. Firstly, Adrian ${ }^{24}$ shows how the uncertainty of instantaneous pixel displacements may be given by Equation 6 where $\sigma_{\Delta x}, \mathbf{M}$ and $\Delta t$ are the potential error in particle location, magnification and inter-frame time respectively. For the presented work, this is found to be approximately $5 \%$. In addition, due to the sheet thickness, perspective error needs to be considered, particularly approaching the edge of the field-of-view due to through-plane velocities. Rafel et. al. ${ }^{25}$ explains how this may be calculated given geometry of the set-up leading to Equation $7^{26}$ where $u_{k}$ is the throughplane velocity and $\alpha$ is the viewing angle. In the presented work, this is a maximum of $3.8 \%$ at the edge of the fieldof-view. Combining these errors in a Pythagorean manner indicates a total error of $6.3 \%$.

$$
\begin{gathered}
\sigma_{u}=\frac{\sigma_{\Delta x}}{M \Delta t} \\
u_{i, \text { true }}=u_{i, \text { meas }}-u_{k} \tan \alpha
\end{gathered}
$$

\section{Ensemble average flow fields}

Initial insight into the flow behaviour under asymmetric valve strategies may be gained by studying the ensemble average vector fields, presented in Figure 5. Changing the MVL can be seen to drastically affect the behaviour and structure of the in-cylinder flow. Figures 5(a), 5(b) \& 5(c) exhibit flow dominated by a counter-rotating vortex pair. The vortex center locations have been identified using an algorithm that finds the peaks of in-plane vorticity. They are highlighted by a cross in these fields. The pair is initially centered in the $\mathrm{x}$-direction within the cylinder with a vortex approximately horizontally under each intake valve as one would expect with symmetrical valve lift, exhibiting typical flow structure for a pent roof, flat piston design. With decreasing MVL the vortex located under the fixed MVL intake valve becomes more dominant due to the increased airflow through this valve.

The cases with least MVL, $0 \%$ and 20\%, Figures 5(f) \& 5(e) respectively show evidence of a single dominant vortex structure. Interestingly, this is located centrally in the $20 \%$ MVL case, but slightly to the positive $\mathrm{x}$-direction for the $0 \%$ lift case. Similarly, there is slight asymmetry seen in the $100 \%$ MVL case which can be seen to bias towards this direction. This is thought to be due to the intake port plenum feed. While there is significantly less flow through the variable MVL valve in the $20 \%$ case compared to the higher MVL cases, the increased velocity of the flow appears to centralise the vortex, resulting in a well defined ensemble field. However in the $0 \%$ MVL case, the ensemble average vortex center is not so precisely defined, suggesting the behaviour is less stable. This is further explored in subsequent analysis presented in the coherent field analysis section.

The 40\% MVL case, Figure 5(d) is unique amongst those conditions tested in that it cannot be categorised into one or two vortex structure flow. There appears a well defined, dominant vortex towards the negative $\mathrm{x}$-direction, while in the positive direction there is no clearly defined vortex location, rather a region in which vortices may be located (highlighted by a rectangle). This is suggestive of unstable behaviour and is further explored in the analysis of the decomposed velocity fields.

With regards to flow velocities, there is an increase of the spatial average velocity of $12.7 \mathrm{~m} / \mathrm{s}$ in the $100 \% \mathrm{MVL}$ condition to $27.2 \mathrm{~m} / \mathrm{s}$ in the $0 \% \mathrm{MVL}$ condition due to the higher port exit velocity as a result of reduced effective valve area in the lower lift cases and nominally constant mass flow rate. This is in similar fashion to other studies featuring reduced lift ${ }^{27 ; 28}$.

\section{Vector field separation}

A technique has been developed to separate the raw captured velocity fields into coherent and stochastic turbulent constituent fields through the use of cross-correlation. It is assumed that the flow consists of large scale structures, evident in all captured fields, with their size and location 


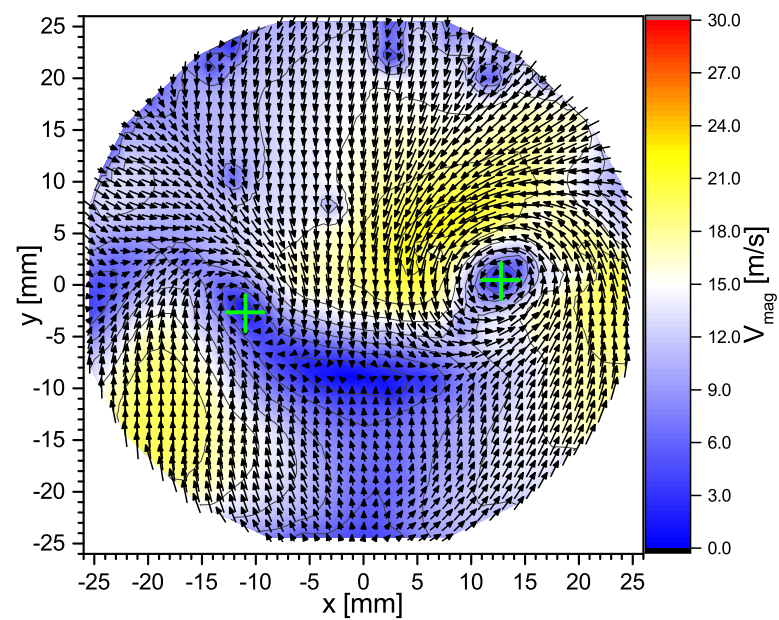

(a) $100 \% \mathrm{MVL}$

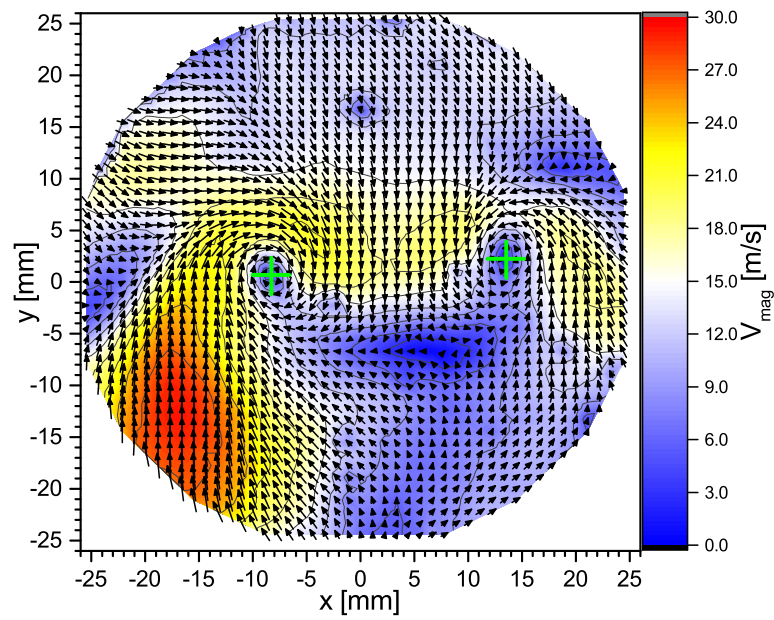

(c) $60 \% \mathrm{MVL}$

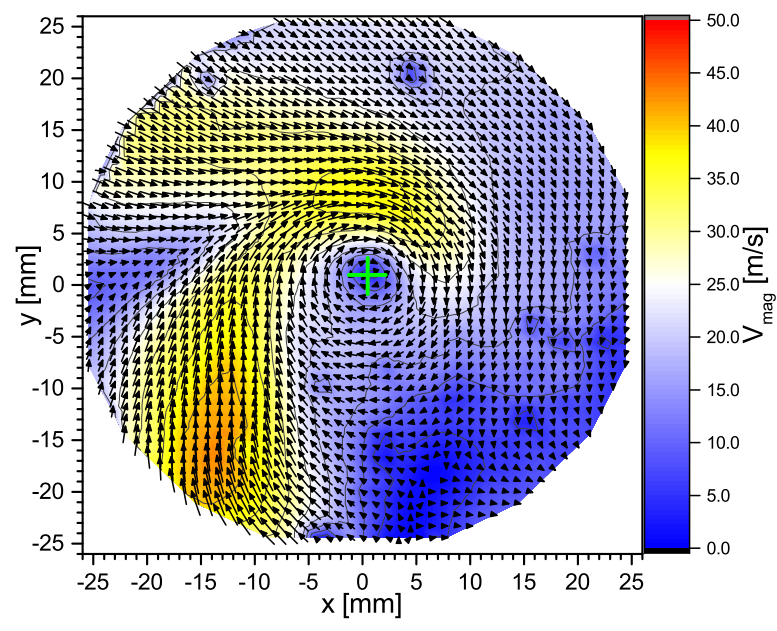

(e) $20 \% \mathrm{MVL}$

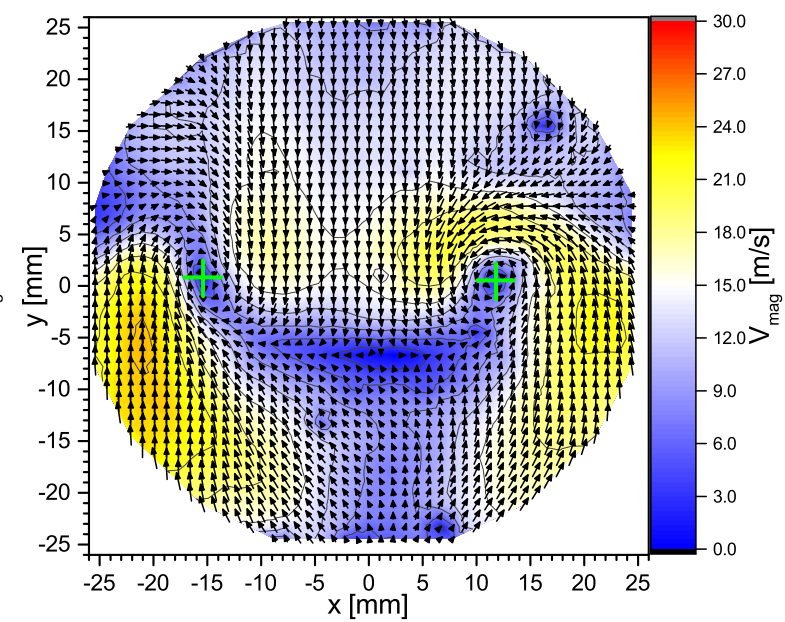

(b) $80 \% \mathrm{MVL}$

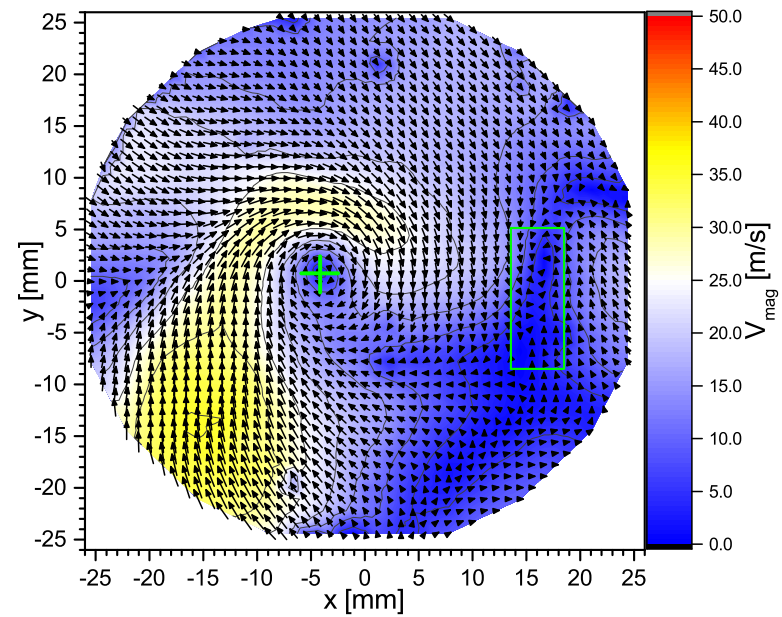

(d) $40 \% \mathrm{MVL}$

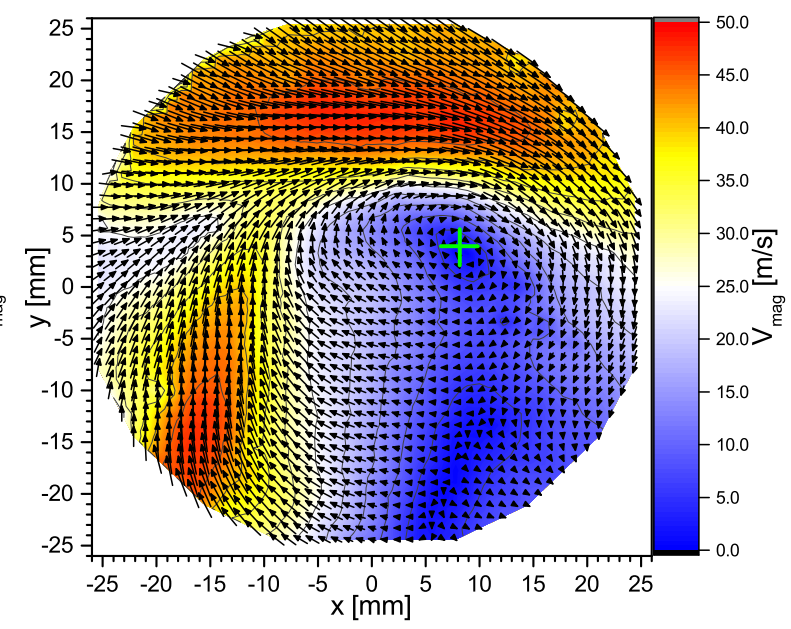

(f) $0 \% \mathrm{MVL}$

Figure 5. Ensemble average flow fields for the tested range of MVL conditions at $75^{\circ} \mathrm{CA}$ (Dominant vortices centers marked, note changing scale at $40 \% \mathrm{MVL}$ )

subject to cyclic variation. In addition, the flow features smaller scale stochastic turbulence, which may not ordinarily be resolved using non-time resolved measurement methods.

The POD technique discussed previously may identify the most energetic modes relating to coherent structures as lower order modes. While the higher order modes represent stochastic turbulence, however, it is not clear where the cutoff lies.

In repeated PIV experiments of the same configuration, it is expected that the same coherent structures should be evident in velocity fields captured from both experiments. It therefore follows that POD analysis carried out would 
identify the same lower order modes, representing the energetic, coherent motion. Conversely, in having stochastic properties, there would be little or no correlation between the higher order modes. Therefore, there exists a threshold after which the two sets of POD spatial modes would no longer show evidence of correlation.

This is defined as the cut-off mode. Spatial and temporal modes preceding this are used for the reconstruction of the coherent velocity fields. Modes above the cut-off are used in the reconstruction of the turbulence present.

The presented method uses a sufficient number of samples (velocity fields) from a single experiment to split into two sets. A 'sufficient number' of samples is dependent on the nature of the flow fields and is assessed as part of the algorithm based on the convergence of velocity statistics. This is described in the supplemental material section. A potential problem introduced by the use of a single large dataset is that of drift, where the test conditions may change steadily over the duration of the test. It is therefore good practice that the sampling order of captured data fields be randomised. However, there was no evidence of drift over the testing period in the presented work.

\section{Correlation}

Correlation functions are a branch of two-point statistics that give a quantifiable measure of similarity between a velocity at one point in space and time with another. It is based on the normalised form of covariance. The generalised form may be applied to a sequence of velocity fields, or in the case presented, two sets of vector fields, set A \& B. In this case, Equation 8 allows cross-correlation of each vector field from set A with its corresponding field from set B.

$$
R_{i i, A B}=\frac{\overline{u_{i}(x)_{A} u_{i}(x)_{B}}}{\sqrt{\overline{u_{i}^{2}(x)_{A}}} \sqrt{\overline{u_{i}^{2}(x)_{B}}}}
$$

\section{Algorithm description}

The entire (randomly ordered) data-set is split into set A \& B with POD carried out independently on each set using LaVision DaVis 8.2 software, each producing a set of spatial and temporal modes.

Each spatial mode from set A, $P O D_{A}$ is correlated against the corresponding mode from $P O D_{B}$ according to Equation 8. As well as the corresponding mode, it is also correlated with the 10 surrounding neighbouring modes $(+/-5)$ as shown in Figure 6 where $m_{A} \& m_{B}$ are spatial POD modes from set $\mathrm{A}$ and $\mathrm{B}$ respectively. This allows for modest re-ordering of the dominant modes which can occur, particularly for paired modes of similar energy. It

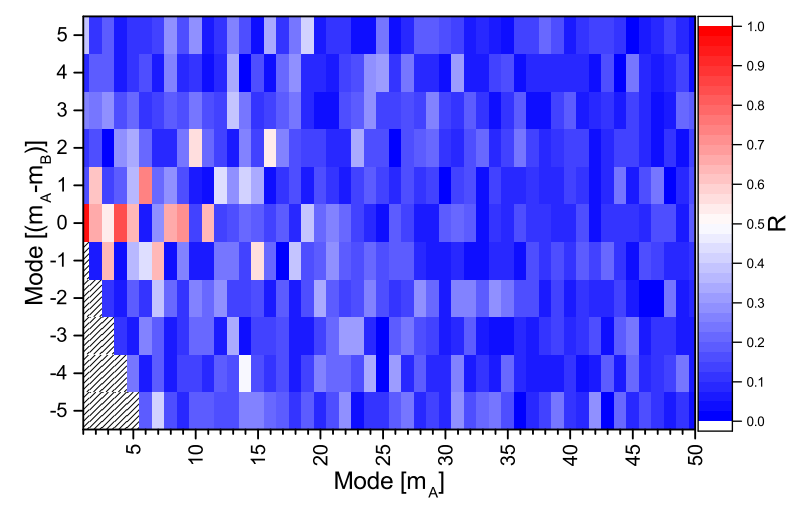

Figure 6. Cross-correlation of $\mathrm{POD}_{A}$ modes with corresponding and surrounding modes from $\mathrm{POD}_{B}$

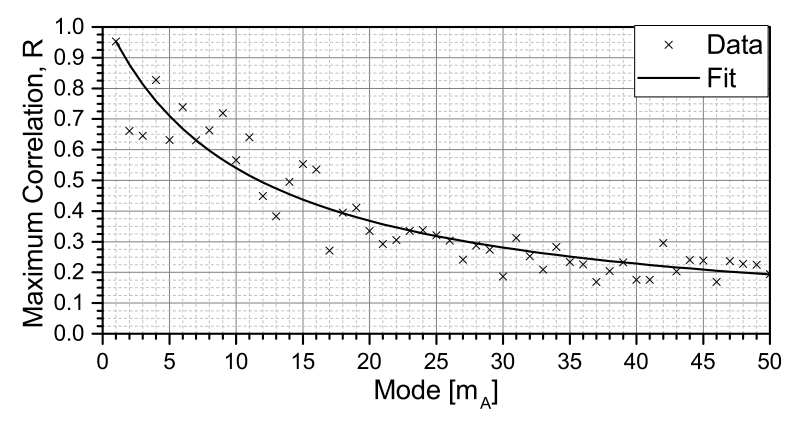

Figure 7. Maximum correlation at each mode with best fit

is often found such pairing occurs because it effectively captures eddy transport when the paired modes are added after scaling with their anti-phase temporal coefficients. This in general results in some modes from set A exhibiting higher correlation with a differing modes from set B. For example, this is evident in mode 6 of set $A$ in Figure 6 which has the largest correlation with mode 5 of set $\mathrm{B}$.

The maximum correlation at each set A mode is selected as shown in Figure 7 and the modulus is taken to nullify the effect of a negative correlation coefficient. This accounts for the situation where a spatial mode may be generated with velocity of opposite sign whose corresponding temporal coefficients would also be of opposite sign. This frequently occurs as the two situations provide equally efficient representation. A fit is obtained from the points and the mode at which the correlation is below 0.5 is considered the cutoff mode. This cut-off whilst appearing some-what arbitrary, was found to give comparable results to other methods investigated such as LES filtering. Within the proposed method, this cut-off is the only tunable parameter.

Each of the two sets, $P O D_{A}$ and $P O D_{B}$ are reconstructed with all modes lower than the cut-off representing the coherent fields, and those above representing the turbulent fields. Finally, the reconstructed vector fields from each set, $P O D_{A}$ and $P O D_{B}$ are recombined in their original sample order to give sets of the original size. An example 


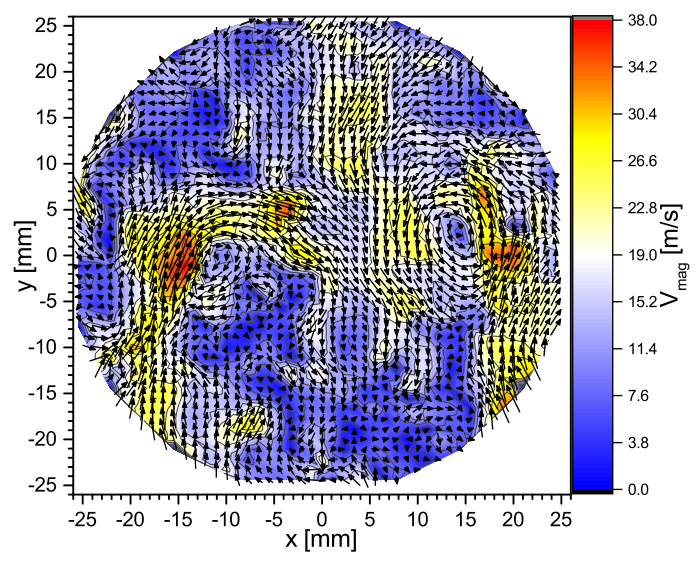

(a) Raw field

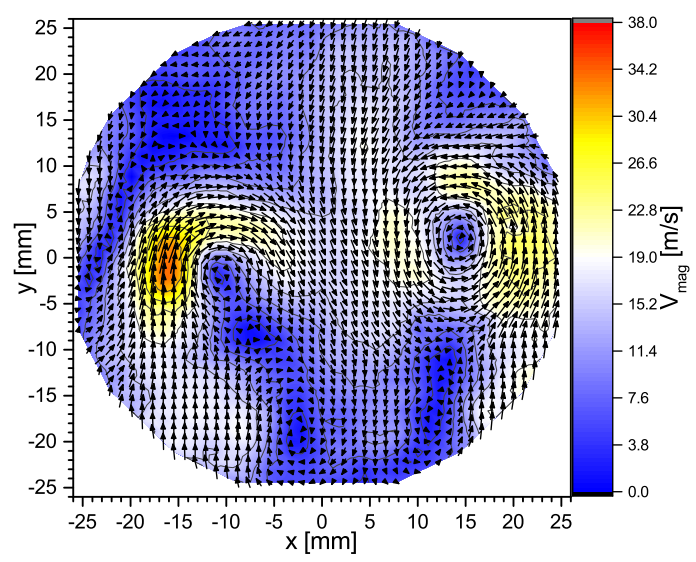

(b) Coherent field

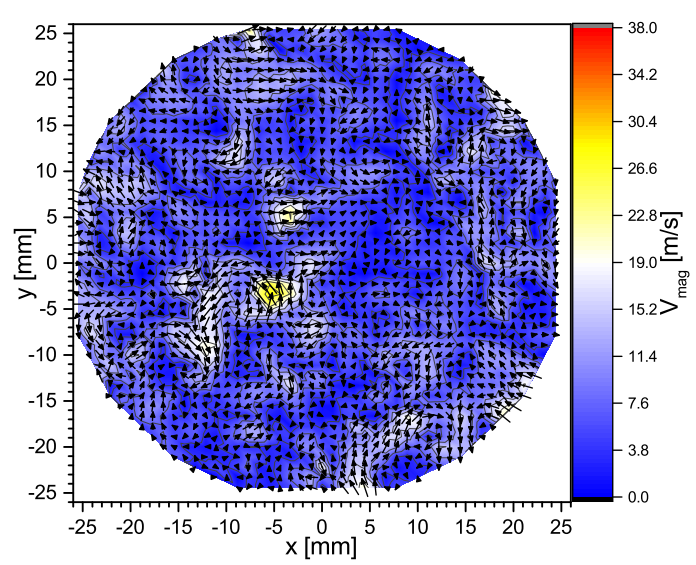

(c) Turbulent field

Figure 8. Original instantaneous vector field example with coherent and turbulent constituent fields (100\% MVL, $\left.70^{\circ} \mathrm{CA}\right)$

of the raw, coherent and turbulent parts for an instantaneous field are presented in Figure 8.

\section{Analysis of constituent fields}

\section{Coherent field analysis}

In a previous section, studying the ensemble average velocity fields revealed less well-defined vortex locations in both the 0\% MVL and 40\% MVL cases. By analysing the coherent

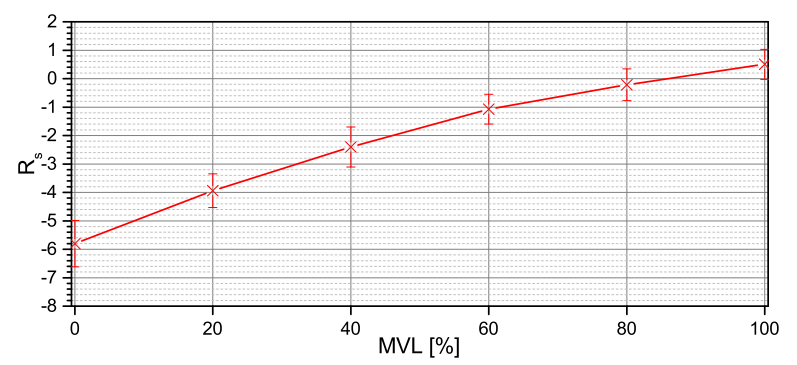

(a) Swirl ratio

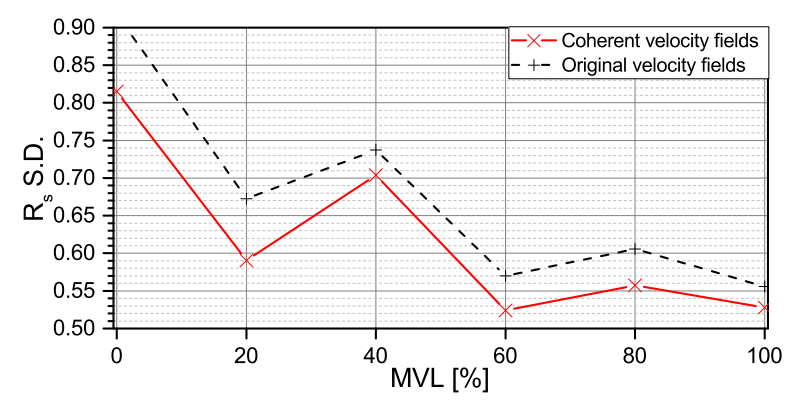

(b) Standard deviation of swirl ratio

Figure 9. Mean swirl ratio and standard deviation at $75^{\circ} \mathrm{CA}$

constituent, a clearer assessment of the stability and cyclic behaviour may be obtained.

It has been seen that the large scale flows are significantly impacted by the variation of MVL. The overall structure and cyclic variation may be quantified using the swirl ratio, $R_{s}$ according to Equation 9 where $\omega_{s}$ and $N$ are swirl angular velocity and engine speed respectively. Further, the cyclic variation is evident in the variation of the swirl ratio, evaluated on individual coherent velocity fields, presented in Figure 9(a). This mean value is found to be equal to the swirl ratio calculated from the ensemble average in each case. A dashed line is included in Figure 9 to represent the standard deviation of swirl ratio calculated from the original velocity fields. The clear trend in swirl ratio shows that valve lift asymmetry increases the magnitude of overall swirl ratio. Note, in this case, swirl ratio is negative due to the direction of rotation.

$$
R_{s}=\frac{\omega_{s}}{2 \pi N}
$$

Figure 9(b) more clearly presents the standard deviation of swirl ratio for each MVL condition. There is evidence of increased variation in the $0 \%$ MVL and $40 \% \mathrm{MVL}$ conditions as a result of the variation of the vortex center locations between cycles. It is interesting to note that the mean value presented in Figure 9(a) is exactly equal to that calculated directly from the ensemble average flow fields because the effect of the turbulence on the average swirl ratio should be negligible. The standard deviation calculated from the original data also follows a similar trend to that calculated 


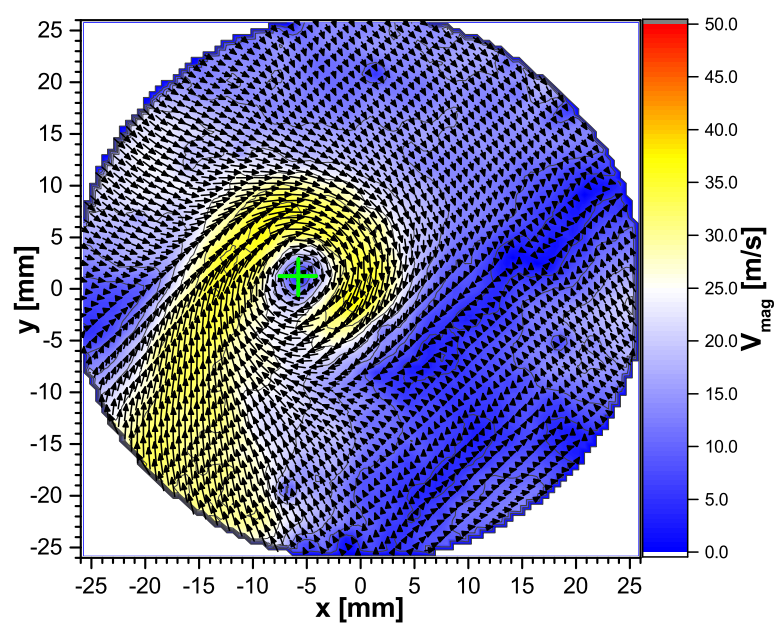

(a) One defined vortex

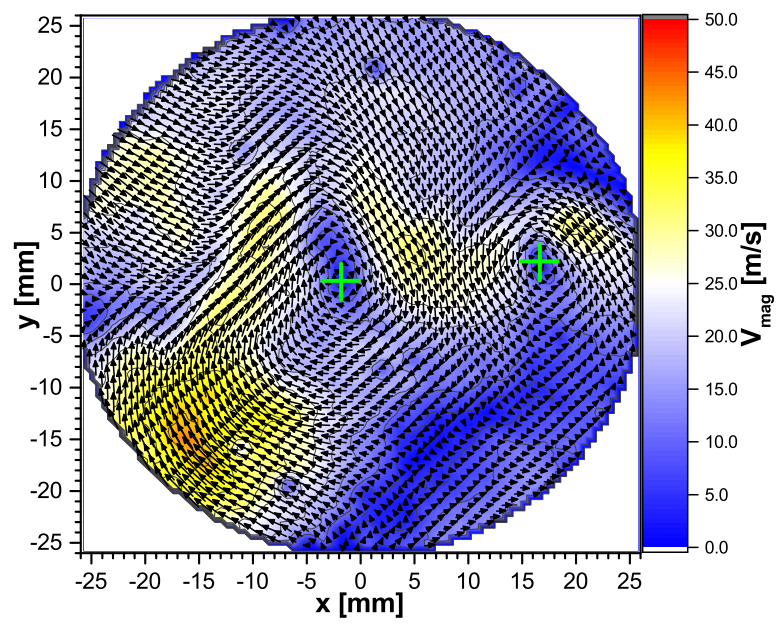

(b) Two defined vortices

Figure 10. Examples of coherent constituents of instantaneous flow fields in $40 \% \mathrm{MVL}$ case $\left(75^{\circ} \mathrm{CA}\right)$

from the coherent fields (Figure 9), but is increased due to the extra variation arising from the included turbulence in the original fields.

Figure 11 presents the spatial distribution of vortex centers for each of the MVL test conditions. As expected, in the $20 \%, 60 \%, 80 \%$ and $100 \%$ MVL conditions (Figures 11(e),11(c),11(b) \& 11(a) respectively), there is reasonably little spread of the vortex centers, in agreement with the analysis thus far. In the $40 \%$ MVL case (Figure 11(d)), there is strong evidence of a switching behaviour between a single vortex and vortex pair structures. There is a lower count of vortex centers located in the positive $\mathrm{x}$-direction region showing only approximately $25 \%$ of cycles have the two vortex structure, and a relatively large spread of vortex center locations for the other vortex. Figure 10 shows two examples of the coherent constituents of instantaneous flow fields from the $40 \%$ MVL case, clearly demonstrating an example of a cycle with a single vortex structure (Figure 10(a)) and a cycle with two vortices (Figure 10(b)).
Also, the increased cyclic variability in the 0\% MVL case is further evident in Figure 11(f) where there is a large spread of vortex center locations. Identifying the cyclic variation in flow behaviour is essential to the development of combustion engines as there is a strong link between the variation of in-cylinder flow and subsequent variation in combustion performance $^{29}$.

\section{Turbulent field analysis}

Analysis of the turbulent constituent fields allows distributions of TKE to be determined without influence of cyclic variation. This section describes analysis carried out using reconstructions from only the POD modes representative of turbulence as previously defined. Figure 12 presents the spatial distribution of turbulent kinetic energy in the $100 \%$ MVL case. The TKE is distribution reasonably uniformly throughout the plane, and is similar in each of the other MVL cases tested, albeit with differing magnitudes. Therefore a spatial average is taken for each case and presented in Figure 13 together with spatially averaged TKE for the coherent and raw fields. The TKE shows a clear increase with increasing valve asymmetry. One source of turbulent kinetic energy production during the intake stroke is the interaction between the swirling in-cylinder motion and the high velocity intake jet(s), where velocity gradients are most significant. As MVL is reduced, the jet velocities and therefore velocity gradients are increased, resulting in an increase in TKE.

It was stated at the outset of this study, that measured TKE may be an overestimation by as much as $300 \%{ }^{2}$, as it contains contributions from both cyclic variation and turbulence. In the cases presented, it may be shown that the TKE of the stochastic velocity fields is $66 \%$ of the total TKE. Further, in Figure 13 it has been shown how the total TKE calculated from the original fields is equal to the sum of TKE calculated from both the coherent and turbulent fields, leading to Equation 10. Where $u, U^{*} \& u^{\prime}$ are original, coherent and turbulent velocities as decomposed by Equation 2 and are calculated from the three separated vector field sets. The relationship may be proven mathematically provided the mean of coherent and turbulent velocity fields are zero.

$$
T K E_{u}=T K E_{U^{*}}+T K E_{u^{\prime}}
$$

Applying the described methodology to the flow fields of each of the MVL test conditions independently results in a single cut-off mode calculated for each dataset. This is found to be between 9 - 11 for all conditions (values given in Table 4), suggesting a similar break-down of fluctuation energy 


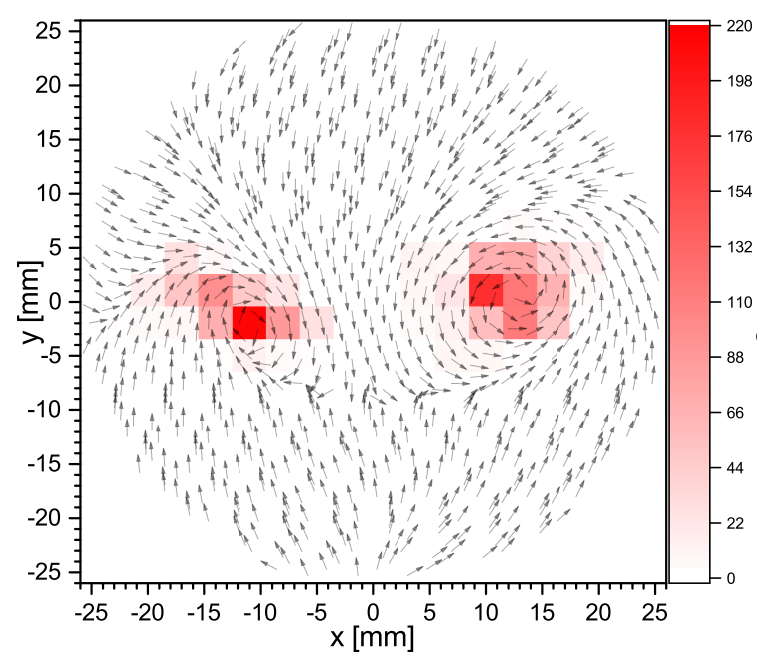

(a) $100 \% \mathrm{MVL}$

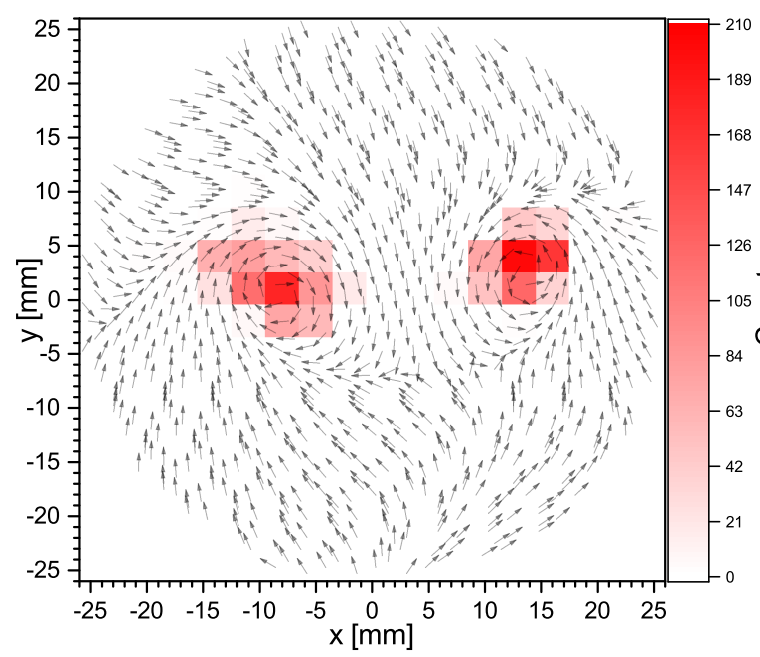

(c) $60 \% \mathrm{MVL}$

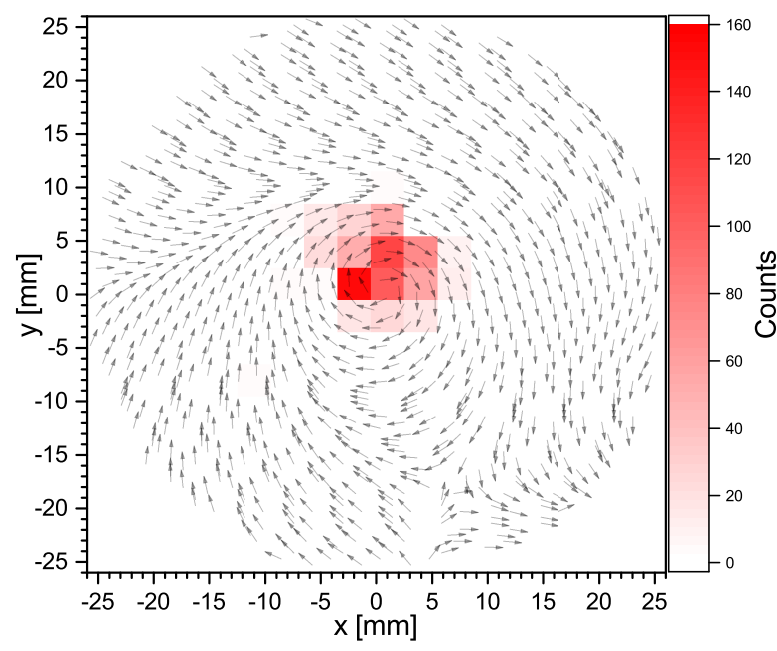

(e) $20 \% \mathrm{MVL}$

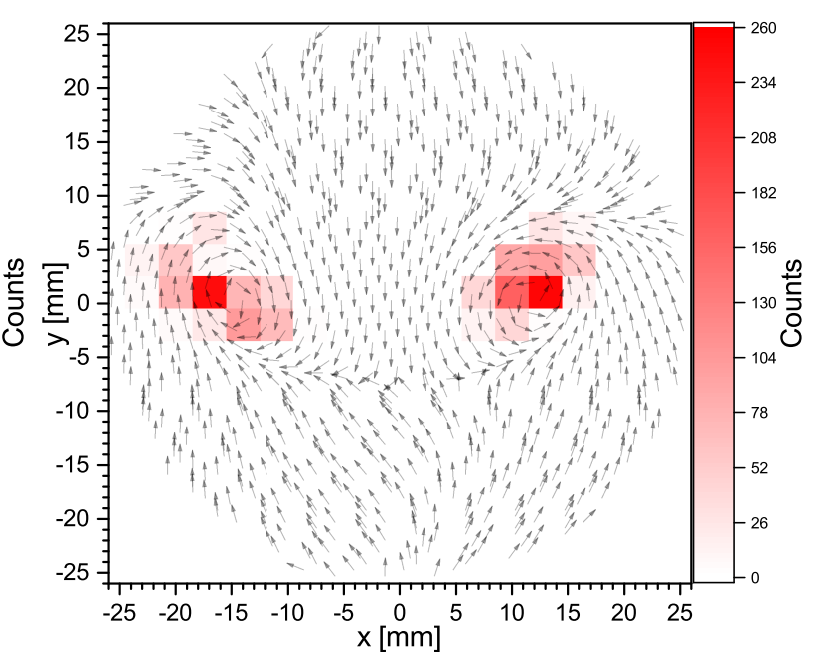

(b) $80 \% \mathrm{MVL}$

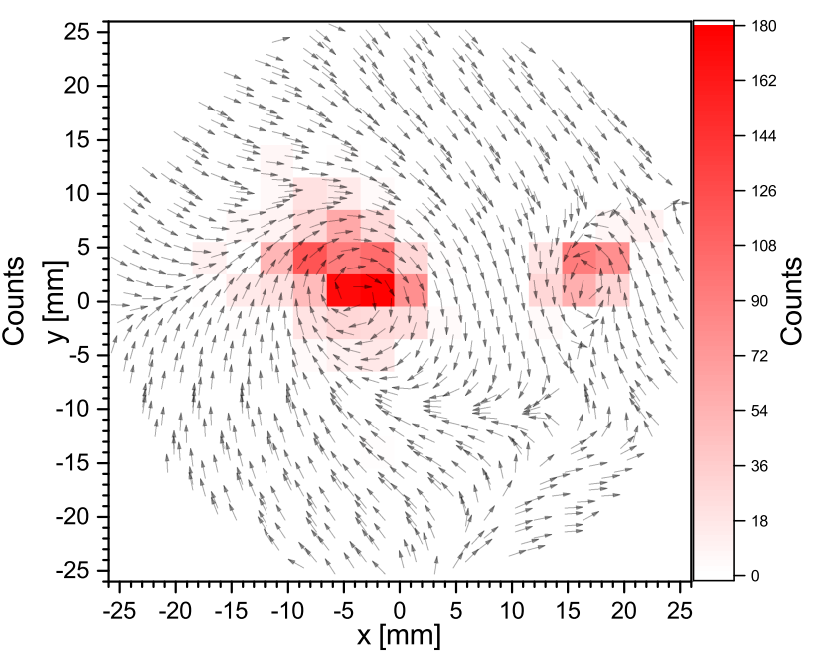

(d) $40 \% \mathrm{MVL}$

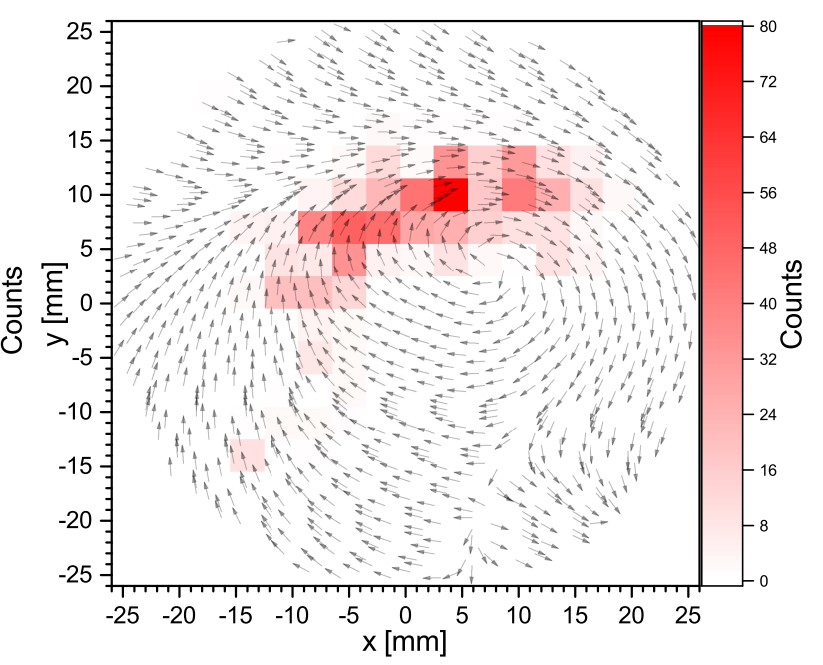

(f) $0 \% \mathrm{MVL}$

Figure 11. Vortex center distributions $\left(9 \mathrm{~mm}^{2}\right.$ bins) with ensemble average velocity field background

from the cyclic variation and turbulence contributions in each case.

\section{Summary}

The effects of asymmetric valve strategies were investigated through PIV measurements and the use of a novel technique for analysing the resulting velocity fields. The main findings may be summarised as follows: 


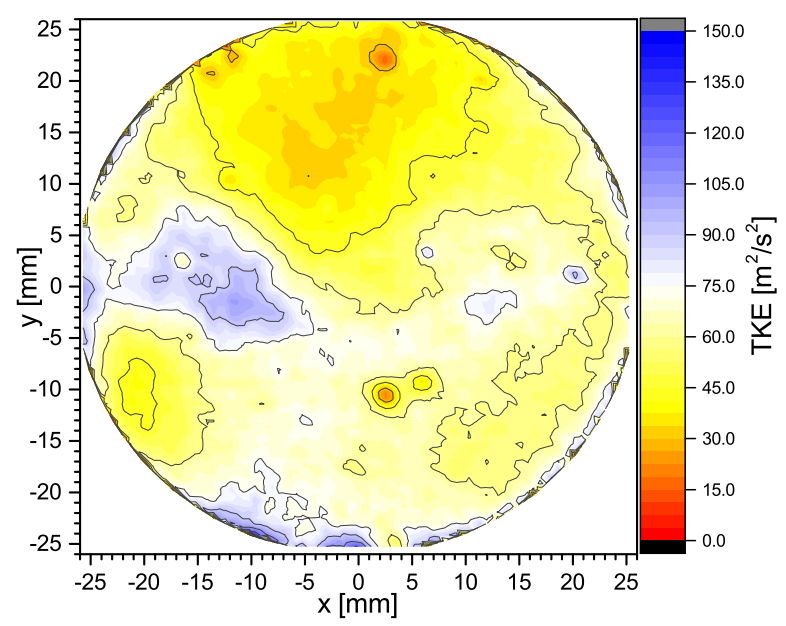

Figure 12. Turbulent kinetic energy distribution at $100 \% \mathrm{MVL}$ $\left(75^{\circ} \mathrm{CA}\right)$

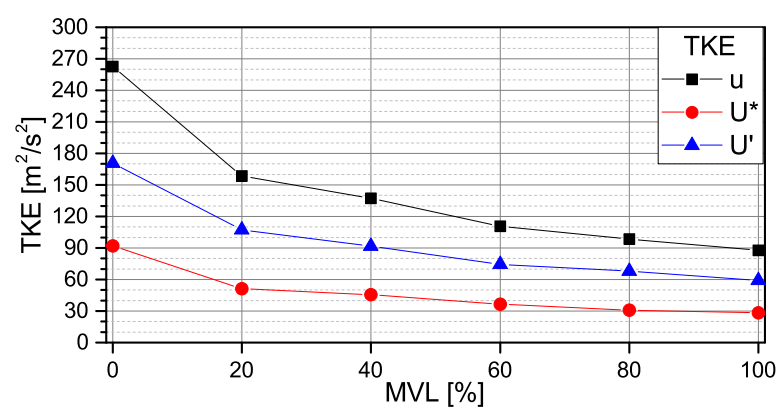

Figure 13. Spatially averaged TKE for range of MVL conditions $\left(75^{\circ} \mathrm{CA}\right)$

Table 4. Cut-off modes

\begin{tabular}{cc}
\hline MVL scale & Cut-off mode $\left[75^{\circ}, 80^{\circ}, 85^{\circ}\right]$ \\
\hline 0 & $11,11,11$ \\
20 & $9,11,10$ \\
40 & $10,10,10$ \\
60 & $10,9,11$ \\
80 & $10,9,11$ \\
100 & $11,11,11$ \\
\hline
\end{tabular}

(i) Correlation of spatial POD modes between independent data sets provides a robust technique for identifying the modes representative of large scale coherent motion with strong correlation and those representative of turbulence with weak or no correlation. The technique is more robust than selecting an arbitrary cut-off cumulative energy level as commonly employed.

(ii) Symmetric or close to symmetric valve strategies provide a flow structure dominated by a counterrotating vortex pair in the swirl plane, while asymmetric strategies may be dominated by a single vortex. It was found that the switch between the two occurs at the $40 \%$ MVL condition. This is responsible for an increase in the cyclic variability of swirl ratio in this condition. (iii) An increase in the overall swirl number magnitude is observed with decreasing MVL as the vortex centers and relative strengths change. The symmetric valve lift case, $100 \%$ MVL, results in a swirl ratio of 0.5 , which changes to 6.0 for the single valve condition, $0 \%$ MVL. Similarly, there is an increase in $\mathrm{TKE}_{u^{\prime}}$ as the MVL is reduced.

(iv) In separating the fluctuating velocity components into a cyclic varying component and truly stochastic component, it was determined that the stochastic fluctuations responsible for enhancing mixing and flame speed were only $66 \%$ of the total TKE calculated from the ensemble statistic. Standard ensemble turbulence statistics can therefore be misleading since the TKE associated with the cyclic variation component cannot enhance the mixing of flame speed within any one individual cycle. This is consistent with the review of work in this area contained in Stone ${ }^{2}$.

\section{Acknowledgements}

The authors gratefully acknowledge of the support of Group Lotus for the Optical SCORE Engine and the Active Valve Train system.

\section{Declaration of conflicting interests}

The authors declare that there is no conflict of interest.

\section{Funding}

The authors received no financial support for the research, authorship and/or publication of this article.

\section{References}

1. Baby X, Dupont A, Ahmed A et al. A New Methodology to Analyze Cycle-to-Cycle Aerodynamic Variations. SAE Technical Paper 2002; (2002-01-2837). DOI:10.4271/200201-2837.

2. Stone R. Introduction to internal combustion engines. 4th ed. Basingstoke: PALGRAVE MSCMILLAN, 2012. ISBN ISBN 9780230576636 (hbk.);ISBN 023057663X. DOI: 10.1017/CBO9781107415324.004. arXiv:1011.1669v3.

3. Joo SH, Srinivasan KK, Lee KC et al. The behaviour of smalland large-scale variations of in-cylinder flow during intake and compression strokes in a motored four-valve spark ignition engine. International Journal of Engine Research 2004; 5(4): 317-328. DOI:10.1243/146808704323224222.

4. Liu D, Wang T, Jia M et al. Cycle-to-cycle variation analysis of in-cylinder flow in a gasoline engine with variable valve lift. Experiments in Fluids 2012; 53(3): 585-602. DOI: 10.1007/s00348-012-1314-4. 
5. Wang T, Liu D, Tan B et al. An investigation into in-cylinder tumble flow characteristics with variable valve lift in a gasoline engine. Flow, Turbulence and Combustion 2014; 94(2): 285304. DOI:10.1007/s10494-014-9562-4.

6. Li Y, Zhao H, Leach B et al. Characterization of an incylinder flow structure in a high-tumble spark ignition engine. International Journal of Engine Research 2004; 5(5): 375-400. DOI:10.1243/1468087042320924.

7. Ölçmen S, Ashford M, Schinestsky P et al. Comparative Analysis of Velocity Decomposition Methods for Internal Combustion Engines. Open Journal of Fluid Dynamics 2012; 02(03): 70-90. DOI:10.4236/ojfd.2012.23008.

8. Berkooz G, Holmes P and Lumley J. The proper orthogonal decomposition in the analysis of turbulent flows. Annu Rev Fluid Mech 1993; 25(1971): 539-575. DOI: 10.1146/annurev.fl.25.010193.002543.

9. Lumley J. The Structure of Inhomogeneous Turbulent Flows. Atmospheric Turbulence and Radio Wave, Nauka 1967; : 166178.

10. Druault P, Guibert $\mathrm{P}$ and Alizon F. Use of proper orthogonal decomposition for time interpolation from PIV data: Application to the cycle-to-cycle variation analysis of in-cylinder engine flows. Experiments in Fluids 2005; 39(6): 1009-1023. DOI:10.1007/s00348-005-0035-3.

11. Cosadia I, Borée J, Charnay G et al. Cyclic variations of the swirling flow in a Diesel transparent engine. Experiments in Fluids 2006; 41(1): 115-134. DOI:10.1007/s00348-006-01634.

12. Cosadia I, Borée J and Dumont P. Coupling timeresolved PIV flow-fields and phase-invariant proper orthogonal decomposition for the description of the parameters space in a transparent Diesel engine. Experiments in Fluids 2007; 43(23): 357-370. DOI:10.1007/s00348-007-0338-7.

13. Voisine M, Thomas L, Borée J et al. Spatio-temporal structure and cycle to cycle variations of an in-cylinder tumbling flow. Experiments in Fluids 2011; 50(5): 1393-1407. DOI: 10.1007/s00348-010-0997-7.

14. Zentgraf F, Baum E, Böhm B et al. On the turbulent flow in piston engines: Coupling of statistical theory quantities and instantaneous turbulence. Physics of Fluids (1994-present) 2016; 28(4): 045108. DOI:10.1063/1.4945785.

15. Chatterjee A. An introduction to the proper orthogonal decomposition. Current Science 2000; 78(7): 808-817. DOI: 10.1109/LPT.2009.2020494.

16. Holmes P, Lumley JL and Berkooz G. Turbulence, coherent structures, dynamical systems and symmetry. Cabridge: Cambridge University Press, 1996.

17. Sirovich L. Turbulence and Dynamics of Coherent Structues. Part I: Coherent Structures. Quarterly of Applied Mathematics
1987; 45(3): 561-571.

18. Graftieaux L, Michard M and Grosjean N. Combining PIV, POD and vortex identification algorithms for the study of unsteady turbulent swirling flows. Meas Sci Technol Meas Sci Technol 2001; 12(1201): 1422-1429. DOI:10.1088/09570233/12/9/307.

19. LaVision. Aerosol Generator Product-Manual, 2012.

20. Melling A. Tracer particles and seeding for particle image velocimetry. Measurement Science and Technology 1997; 8: 1406-1416.

21. Adrian RJ and Westerweel J. Particle Image Velocimetry. Cambridge University Press, 2011.

22. Lancaster DR. Effects of Engine Variables on Turbulence in a Spark-Ignition Engine. SAE Technical Paper 1976; (760159). DOI:10.4271/760159.

23. Li Y, Zhao H and Ladommatos N. Analysis of large-scale flow characteristics in a four-valve spark ignition engine. Proceedings of the Institution of Mechanical Engineers, Part D: Journal of Automobile Engineering 2002; 216(June): 923938.

24. Adrian RJ. Dynamic ranges of velocity and spatial resolution of particle image velocimetry. Measurement Science and Technology 1997; 8: 1393-1398. DOI:10.1088/09570233/8/12/003.

25. Raffel M, Willert CE, Wereley ST et al. Particle Image Velocimetry: A Practical Guide, volume 2nd. 2007. ISBN 9783540723073. DOI:10.1097/JTO.0b013e3182370e69. arXiv:1011.1669v3.

26. Dunham D. Unsteady fluid mechanics of annular swirling shear layers. PhD Thesis, Loughborough University, 2011.

27. Battistoni $M$ and Mariani F. Fluid Dynamic Study of Unthrottled Part Load SI Engine Operations with Asymmetric Valve Lifts 2009; DOI:10.4271/2009-24-0017.

28. Stansfield P, Wigley G, Garner C et al. Unthrottled engine operation using variable valve activation: the impact on the flow field, mixing and combustion. SAE Technical Paper Series 2007; (2007-01-1414).

29. Justham T. Cyclic Variation in the Flow Field Behaviour within a Direct Injection Spark Ignition Engine: A High Speed Digital Particle Image Velocimetry Study. PhD Thesis, Loughborough University, 2010.

\section{Supplemental material}

The POD based separation algorithm is built in MATLAB software and first uses a random number generator function to randomly process the velocity fields. This eliminates the issue of drift throughout a large data-set. Although there was no evidence of drift throughout the experiments, it is recognised as a good practice to 
carry out this step. POD modes in set A are therefore calculated from 400 samples randomly selected from the 800 . POD modes of set $\mathrm{B}$ are calculated from the remaining 400 .

The vector fields, sequenced according to their randomly ordered indices are sequentially combined for the calculation of the ensemble average and rms velocity distributions from one field through the entire data-set. The convergence of these values are observed at half the sample size to within $\pm 0.5 \%$ in Figure 14 . If the values have converged by this point, then the data-set is of sufficient size to proceed.

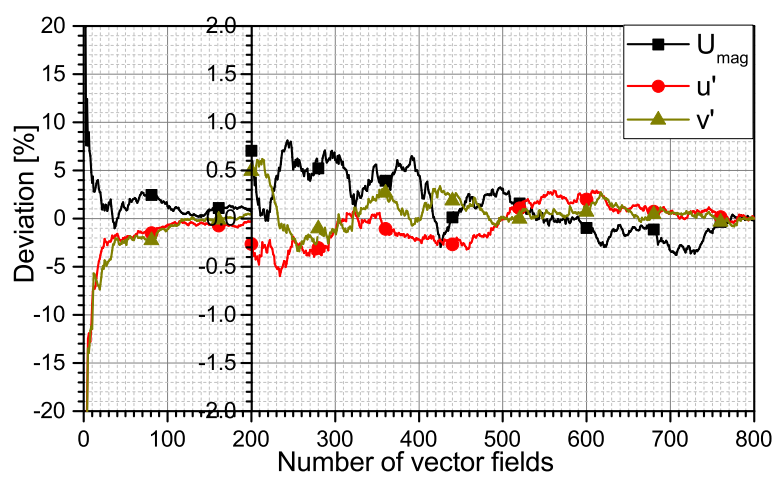

Figure 14. Deviation of mean velocity magnitude $\left(U_{\text {mag }}\right)$ and rms velocities (u' \& v') spatial averages from values calculated from full data set (N.B. deviation scale change at $\mathrm{N}=200$ to + $2 \%)$ 